\title{
A matter of scale
}

\author{
Nanomaterials research must overcome the issue of scalability if it is to make a tangible \\ impact in the real world.
}

One of the tenets of nanoscience is the fact that the physical and chemical properties of matter change with size, and as you reach the nanometre length scale new behaviours start to appear. This is largely the appeal of doing science at this scale. We can now study, manipulate and control nano-objects - even one at a time in many cases - to produce a desired effect or a property that cannot be achieved in the bulk. The rush to the nanoscale is almost ubiquitous in science, because nanoscience offers the unique ability to understand physical and chemical processes at the most fundamental level. In principle, this knowledge should be instrumental to improve and optimize a system under investigation.

But despite these fundamental advances, nanotechnology is confronted with a critical bottleneck. We are still struggling to translate the fundamental advances reported in the scientific literature into tangible technological applications that can be appreciated at the layman's level. The problem is twofold. First, the properties of matter change when scaled up, just like they change when scaled down to the nanoscale; in particular, the level of control that can be exerted at the nanoscale or at the single-object level tends to wane at the meso- and macroscales or when dealing with a large number of objects. And second, industry is reluctant to invest money in developing new large-scale processes for nanomaterial fabrication unless they are guaranteed a sizeable profitable return.

The situation is especially relevant in applied fields, because there is a disconnect between laboratory- and industrial-scale investigation.

It is with this backdrop in mind that, in 2010, the United States Department of Energy created the Materials Engineering Research Facility (MERF) at the Argonne National Laboratories. Now employing about 20 researchers, most of whom have an industrial background, the facility's main objective is to develop a manufacturing process that takes promising materials from the laboratory scale (usually made on the order of milligrams) to the kilogram scale. This quantity is usually enough to get industrial partners interested to pilot their own large-scale production process.

The whole process is carried out in three consecutive stages in which the material production is scaled up by an order of magnitude each time. The researchers make a large number of batches and must ensure that each batch shows consistent performances, and that the performances do not degrade too much with scaling up.

Other considerations that come into play may be the amount of waste and energy consumption, possibility of recycling, material safety, and hazard level during production - all of which affect the final cost of the process. If, at some point, it becomes clear that, either due to poor performances or high cost, a material is unlikely to be suitable for industrial purposes, the scalingup efforts stop. If successful, however, MERF makes available, to a potential licensee, a technology transfer package detailing the process used to make a kilogram of the material. The document is publicly available.

Kris Pupek is a team leader at MERF and has developed successful scaleup processes for dozens of materials, mostly for electrochemical energy storage and energy conversion. He says that, according to his experience,

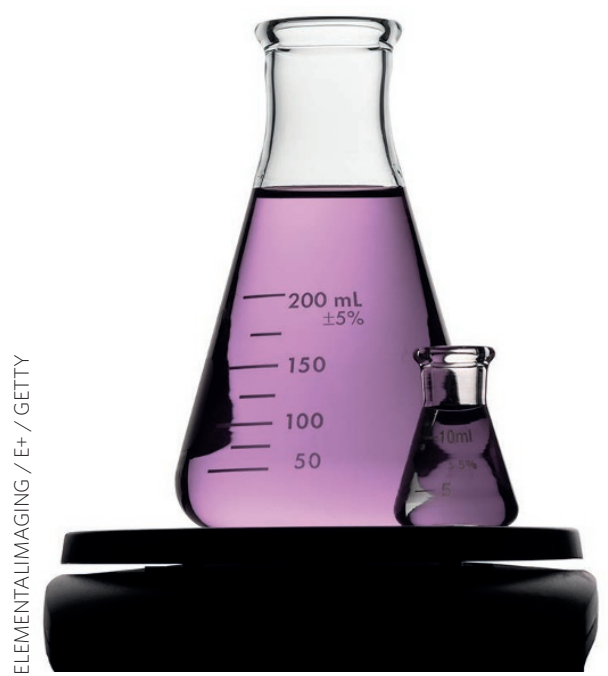

nanomaterials are the trickiest to scale up because of a frequent degradation in performances and batch-to-batch variability. In addition, it is often the case that properties are inconsistent depending on the supplier.

\section{Nanomaterials are the trickiest to scale up because of a frequent degradation in performances and batch-to-batch variability.}

There is therefore a real risk that large portions of applied research in nanomaterials carried out in academia could become irrelevant to the development of future technologies, even though they are often interesting enough to get published in a scientific journal. (Bluesky research is not expected to produce immediate practical applications and it is valuable in a different kind of way.)

Centres such as the MERF should be commended not only for their day-to-day activities, but also, more generally, because they help promote an appreciation for real-world needs in the academic environment. To this end, other, more established, initiatives such as supporting technology transfer, stimulating start-ups, initiating collaborations with industrial partners, and allowing industry to sponsor new centres on campus can be helpful.

Although drawing a sharp line between fundamental and applied research is not so straightforward, principal investigators doing research in an applied field, especially in nanomaterials where scalability is an inherent problem, should take advantage of any of these opportunities and encourage their students to think more in terms of real-world applications. This attitude will maximize chances that, at some point in the future, their science will be relevant at the layman's level.

After all, measuring impact primarily by the number of publications and citations might be limiting in applied research. 